\title{
Tuning the electronic structures and transport coefficients of Janus PtSSe monolayer with biaxial strain
}

\author{
San-Dong Guo, Xiao-Shu Guo and Ye Deng \\ School of Electronic Engineering, Xi'an University of Posts and Telecommunications, Xi'an 710121, China
}

\begin{abstract}
Due to their great potential in electronics, optoelectronics and piezoelectronics, Janus transition metal dichalcogenide (TMD) monolayers have attracted increasing research interest, the MoSSe of which with sandwiched S-Mo-Se structure has been synthesized experimentally. In this work, the biaxial strain dependence of electronic structures and transport properties of Janus PtSSe monolayer is systematically investigated by using generalized gradient approximation (GGA) plus spin-orbit coupling (SOC). Calculated results show that SOC has a detrimental effect on power factor of PtSSe monolayer, which can be understood by considering SOC effects on energy bands near the Fermi level. With $a / a_{0}$ from 0.94 to 1.06, the energy band gap firstly increases, and then decreases, which is due to the position change of conduction band minimum (CBM). It is found that compressive strain can increase the strength of conduction bands convergence by changing relative position of conduction band extrema $(\mathrm{CBE})$, which can enhance n-type $Z T_{e}$ values. Calculated results show that compressive strain can also induce the flat valence bands around the $\Gamma$ point near the Fermi level, which can lead to high Seebeck coefficient due to large effective masses, giving rise to better p-type $Z T_{e}$ values. The calculated elastic constants with $a / a_{0}$ from 0.94 to 1.06 all satisfy the mechanical stability criteria, which proves that the PtSSe monolayer is mechanically stable in the considered strain range. Our works further enrich studies of Janus TMD monolayers, and can motivate farther experimental works.
\end{abstract}

PACS numbers: 72.15.Jf, 71.20.-b, 71.70.Ej, 79.10.-n Keywords: Strain; Spin-orbit coupling; Power factor; Janus TMD monolayers

\section{INTRODUCTION}

After the successful exfoliation of graphene ${ }^{1}$, twodimensional (2D) materials have attracted increasing attention, and lots of $2 \mathrm{D}$ materials have been synthesized experimentally or predicted theoretically, such as TMD, group-VA, group IV-VI and group-IV monolayers ${ }^{2-6}$. It is firstly proposed by Hicks and Dresselhaus in 1993 7,8 that the low-dimensional systems or nanostructures could be potential thermoelectric materials, and the efficiency of thermoelectric conversion can be described by the dimensionless figure of merit ${ }^{9}, Z T=S^{2} \sigma T /\left(\kappa_{e}+\kappa_{L}\right)$, where $\mathrm{S}, \sigma, \mathrm{T}, \kappa_{e}$ and $\kappa_{L}$ are the Seebeck coefficient, electrical conductivity, working temperature, electronic and lattice thermal conductivities, respectively. Many studies of heat transport properties of $2 \mathrm{D}$ materials have been reported such as TMD, orthorhombic group IV-VI and group-VA monolayers ${ }^{10-15}$. In semiconducting TMD monolayers, it is proved that the SOC has important effects on their electronic transport properties ${ }^{16}$. Strain effects on heat transport properties of $2 \mathrm{D}$ materials have been widely investigated. It is found that tensile strain can improve thermoelectric properties of $\mathrm{ZrS}_{2}$ and $\mathrm{PtSe}_{2}$ by enhancing $S^{2} \sigma$ and reducing $\kappa_{L}{ }^{17,18}$. The strain can effectively tune $\kappa_{L}$ for various kinds of $2 \mathrm{D}$ materials, and the $\kappa_{L}$ shows monotonous increase/decrease and up-anddown behaviors ${ }^{19-22}$ with the increasing tensile strain.

Recently, Janus monolayer MoSSe has been successfully synthesised by fully replacing the top S layer with Se, leading to an out-of-plane structural asymmetry ${ }^{23}$. It is found that the piezoelectric performance of monolayer and multilayer Janus TMD MXY ( $\mathrm{M}=\mathrm{Mo}$ or
Email:sandongyuwang@163.com

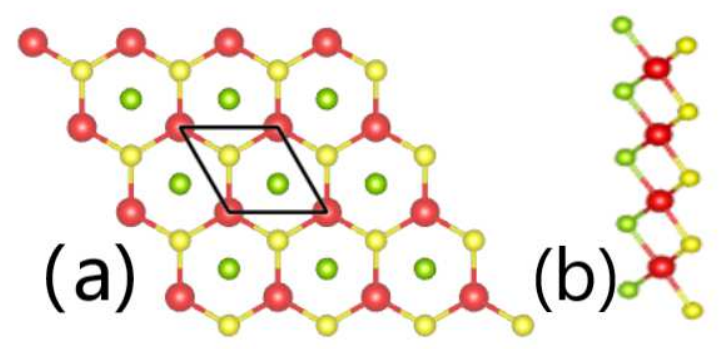

FIG. 1. (Color online) The top view (a) and side view (b) crystal structure of Janus PtSSe monolayer. The Red balls represent $\mathrm{Pt}$ atoms, and the yellow/green balls for $\mathrm{S} / \mathrm{Se}$ atoms.

$\mathrm{W}, \mathrm{X} / \mathrm{Y}=\mathrm{S}$, Se or $\mathrm{Te}$ ) is higher than commonly used $2 \mathrm{D}$ materials ${ }^{24}$. Although the carrier mobility of monolayer MoSSe is relatively low, the one of bilayer or trilayer structures is quite high ${ }^{25}$. In monolayer Janus WSeTe, the intrinsic out-of-plane built-in electric field induces a significant Rashba spin splitting ${ }^{26}$. Janus MoSSe monolayer may be a potential wide solar-spectrum watersplitting photocatalyst ${ }^{27}$, and PtSSe monolayer for photocatalytic water splitting under the visible or infrared light $^{28}$. The optical properties of Janus MoSSe and WSSe monolayers, as well as their vertical and lateral heterostructures, have been investigated by the firstprinciples calculations ${ }^{29}$. The $\kappa_{L}$ of MoSSe/ZrSSe monolayer is predicted, which is much lower than that of the $\mathrm{MoS}_{2} / \mathrm{ZrS}_{2}$ monolayer ${ }^{30,31}$. The biaxial strain dependence of the electronic structures of Janus TMD MXY $(\mathrm{M}=\mathrm{Mo}$ or $\mathrm{W}, \mathrm{X} / \mathrm{Y}=\mathrm{S}, \mathrm{Se}$, or $\mathrm{Te})$ monolayer has been reported $^{32}$, and the strain can effectively tune their elec- 

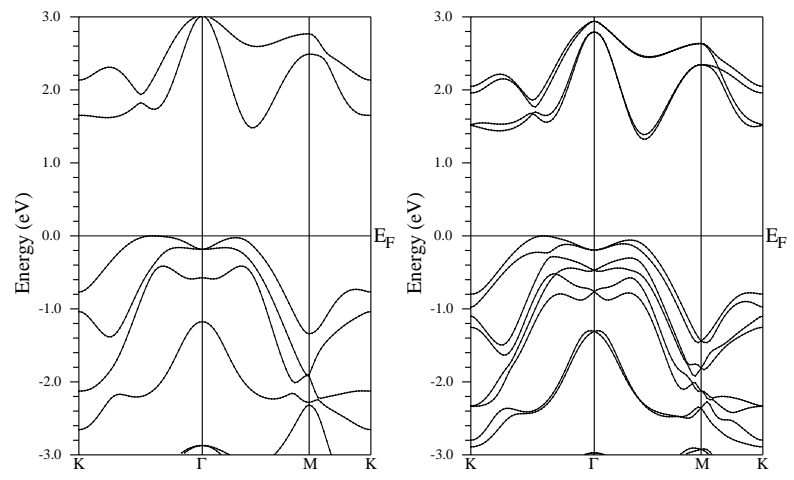

FIG. 2. The energy band structures of PtSSe monolayer using GGA (Left) and GGA+SOC (Right).

TABLE I. For PtSSe monolayer, the lattice constants $a_{0}(\AA)$, the elastic constants $C_{i j}$, shear modulus $G^{2 D}$, Young's modulus $Y^{2 D}$ in $\mathrm{Nm}^{-1}$, Poisson's ratio $\nu$ dimensionless and the gaps with GGA and GGA+SOC.

\begin{tabular}{cccc}
\hline \hline$a_{0}$ & $C_{11} / C_{22}$ & $C_{12}$ & $G^{2 D}$ \\
\hline 3.66 & 77.78 & 22.37 & 27.24 \\
\hline \hline$Y^{2 D}$ & $\nu$ & Gap & Gap-SOC \\
\hline 71.25 & 0.29 & 1.48 & 1.33 \\
\hline \hline
\end{tabular}

tronic structures and transport properties.

In this work, we systematically investigate the biaxial strain dependence of electronic structures and transport properties of Janus PtSSe monolayer by the firstprinciples calculations and Boltzmann equation. It is proved that the SOC can produce important effects on electronic structures and transport properties of PtSSe monolayer. The energy band gap of PtSSe monolayer shows a nonmonotonic up-and-down behavior with $a / a_{0}$ from 0.94 to 1.06 . The compressive strain can tune the position of $\mathrm{CBM}$, and induce flat valence bands, which can obviously affect $\mathrm{S}$. The $\mathrm{n}$-(p-)type $\mathrm{S}$ can be enhanced by applying compressive strain at $a / a_{0}=0.98$ (0.96) point, and then the $Z T_{e}$ can be improved. In considered strain range, the PtSSe monolayer is always mechanically stable.

The rest of the paper is organized as follows. In the next section, the computational details will be given. In the third section, the strain dependence of the electronic structures and transports properties of Janus PtSSe monolayer is shown. Finally, we shall give our discussions and conclusion in the fourth section.

\section{COMPUTATIONAL DETAIL}

Within the density functional theory (DFT) ${ }^{33}$, a fullpotential linearized augmented-plane-waves method is used to investigate electronic structures of PtSSe monolayer, as implemented in the WIEN2k package ${ }^{34}$. The popular GGA of Perdew, Burke and Ernzerhof (GGA-
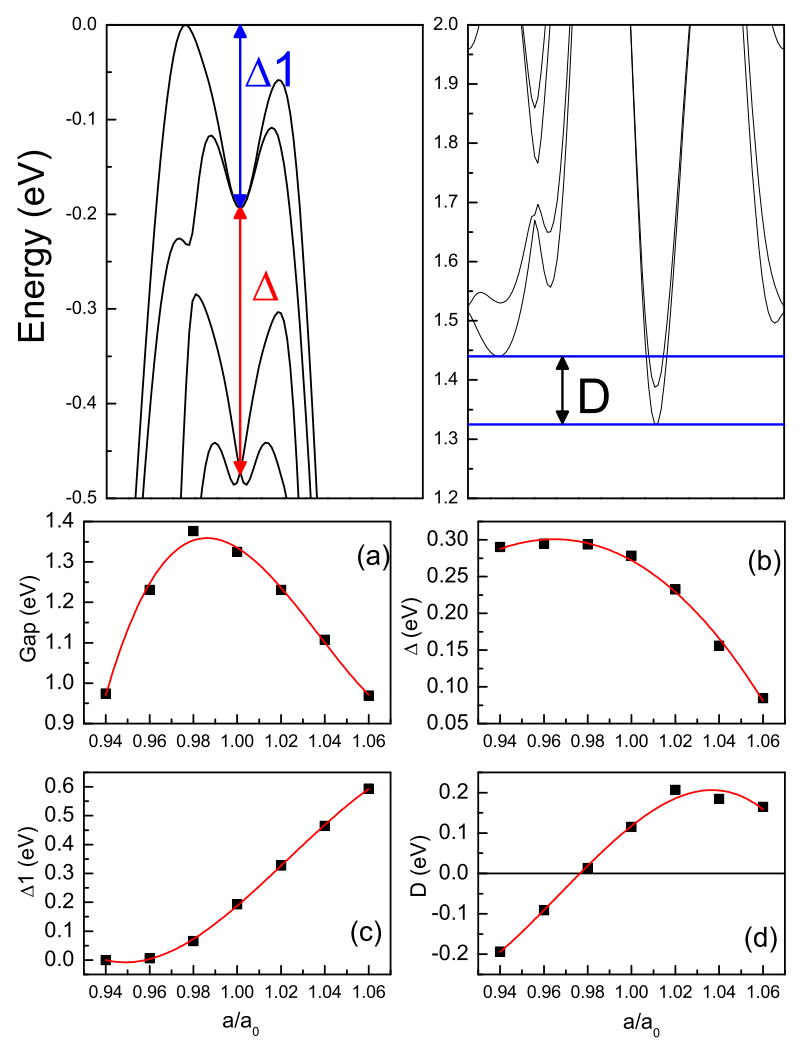

FIG. 3. (Color online)The energy band structures of PtSSe monolayer near the Fermi level. The energy band gap (Gap), $\Delta$ and $\Delta 1$ at high symmetry $\Gamma$ point, and $\mathrm{D}$ as a function of $a / a_{0}$ by using GGA+SOC.

$\mathrm{PBE})^{35}$ is used as the exchange-correlation potential. The internal atomic parameters of PtSSe monolayer are optimized with a force standard of $2 \mathrm{mRy} /$ a.u.. Due to important effects on electronic structure of PtSSe monolayer, the SOC was included self-consistently ${ }^{36-39}$. To attain reliable results, we use a $30 \times 30 \times 1 \mathrm{k}$-point meshes in the first Brillouin zone (BZ) for the self-consistent calculation, make harmonic expansion up to $l_{\max }=10$ in each of the atomic spheres, and set $\mathrm{R}_{\mathrm{mt}} * \mathrm{k}_{\max }=8$. The charge convergence threshold is set as $0.0001|e|$ per formula unit, where $e$ is the electron charge.

By using BoltzTrap ${ }^{40}$ code, the electronic transport coefficients of PtSSe monolayer are performed through solving Boltzmann transport equations within the constant scattering time approximation (CSTA). To achieve the convergence results, the input parameter LPFAC is set to 20. To attain accurate transport coefficients, a $110 \times 110 \times 1 \mathrm{k}$-point meshes is used in the first BZ for the energy band calculation. For 2D material, the calculated $\sigma$ and $\kappa_{e}$ depend on the length of unit cell along z direction ${ }^{41}$, which should be normalized by multiplying $L z / d$ ( $L z$ for the length of unit cell along $\mathrm{z}$ direction, and $d$ for the thickness of $2 \mathrm{D}$ material). However, the $d$ is not well defined like graphene. In this work, the $L z=20 \AA$ is used as $d$. 

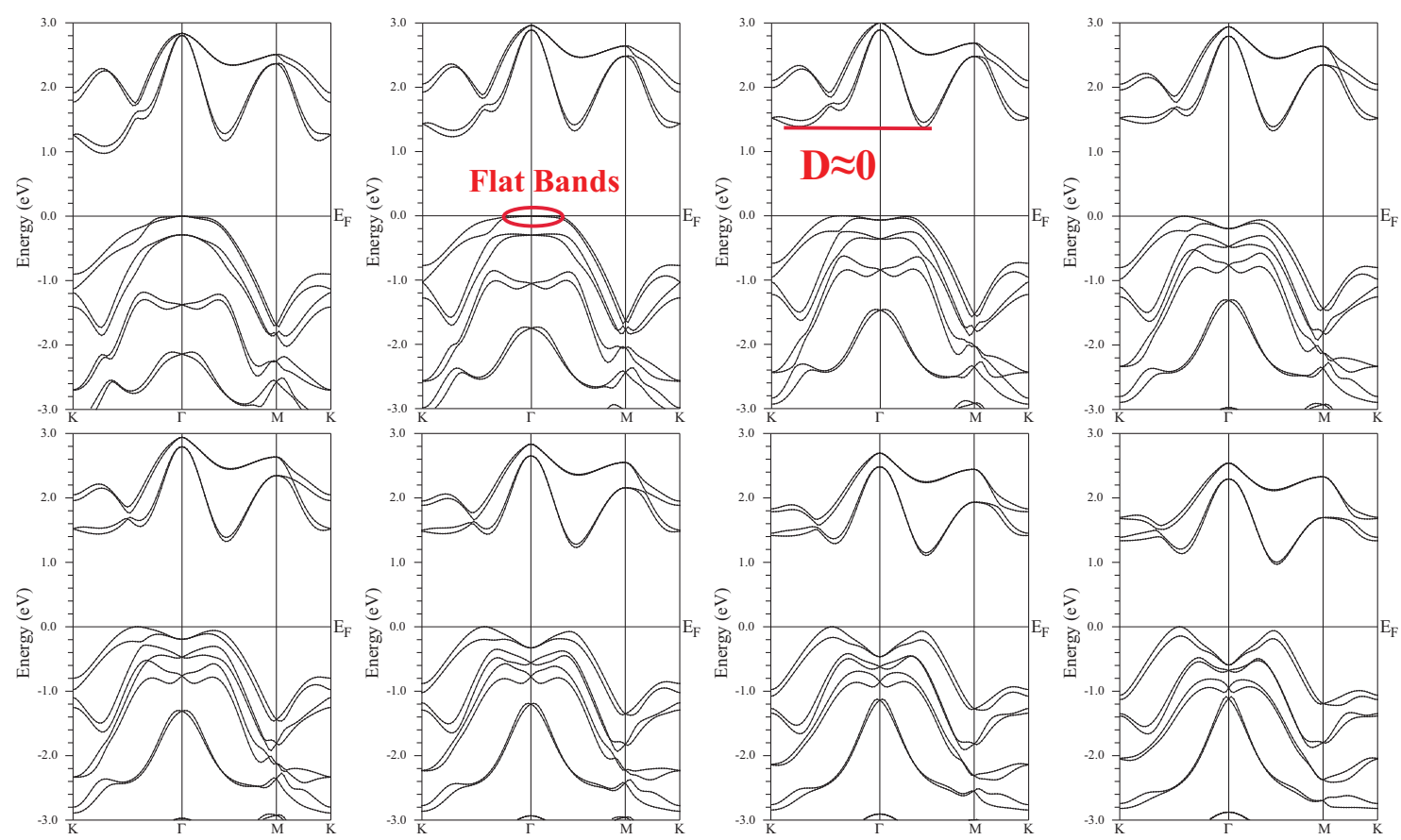

FIG. 4. (Color online)the energy band structures of PtSSe monolayer with $a / a_{0}$ changing from 0.94 to 1.00 (Top) and 1.00 to 1.06 (Bottom) with the interval being 0.02 using GGA+SOC.

\section{MAIN CALCULATED RESULTS AND ANALYSIS}

The structure of Janus PtSSe monolayer is shown in Figure 1, and the three atomic sublayers can be observed with $\mathrm{Pt}$ sandwiched between the $\mathrm{S}$ and Se layers, which is similar to $\mathrm{PtS}_{2} / \mathrm{PtSe}_{2}$ monolayer with the $1 \mathrm{~T}$ phase. Compared with $\mathrm{PtS}_{2} / \mathrm{PtSe}_{2}$, the Janus PtSSe monolayer lacks the reflection symmetry with respect to the central metal Pt atoms, and the symmetry of PtSSe (No.156) is lower than that of the $\mathrm{PtS}_{2} / \mathrm{PtSe}_{2}$ monolayer (No.164). To avoid spurious interaction between neighboring layers, the unit cell of Janus PtSSe monolayer is built with the vacuum region of more than $18 \AA$. The optimized lattice constants of PtSSe (3.66 $\AA$ ) with GGA agree well with previous theoretical values ${ }^{28,42}$, which is between the ones of $\mathrm{PtS}_{2}(3.57 \AA)$ and $\mathrm{PtSe}_{2}(3.75 \AA)^{16}$. Due to hexagonal symmetry, two independent elastic constants $C_{11}=C_{22}=77.78 \mathrm{Nm}^{-1}$ and $C_{12}=22.37 \mathrm{Nm}^{-1} \mathrm{can}$ be calculated, and the $C_{66}=\left(C_{11}-C_{12}\right) / 2=27.24 \mathrm{Nm}^{-1}$, which are very close to previous theoretical values ${ }^{28}$. The $2 \mathrm{D}$ Youngs moduli $Y^{2 D}$, shear modulus $G^{2 D}$ and Poisson's ratios are calculated ${ }^{43}$, and are $71.25 \mathrm{Nm}^{-1}, 22.37$ $\mathrm{Nm}^{-1}$ and 0.29 , respectively. The related data are shown in Table I.

The SOC has important influences on electronic structures of TMD and Janus TMD monolayers, and further influences their electronic transport coefficients ${ }^{16,18,32,44}$. Thus, the SOC is considered for all calculations of Janus PtSSe monolayer. The calculated energy bands for monolayer PtSSe with GGA and GGA+SOC are plotted in
Figure 2. The GGA results show the indirect gap of $1.48 \mathrm{eV}$ with valence band maximum (VBM) along the $\Gamma-\mathrm{K}$ direction and CBM along the $\Gamma-\mathrm{M}$ direction. It is noted that the valence band extrema (VBE) along the $\Gamma-\mathrm{M}$ direction is very close to VBM, and the energy difference is only $0.025 \mathrm{eV}$. When the $\mathrm{SOC}$ is considered, a direct gap of $1.33 \mathrm{eV}$ is observed, and the energy difference between VBE along the $\Gamma-\mathrm{M}$ direction and VBM is $0.058 \mathrm{eV}$, which is larger than one with GGA. It is found that the GGA+SOC gap is smaller than GGA one due to spin-orbital splitting, and the spin-orbit splitting at the $\Gamma$ point $(\Delta$ in Figure 3 ) is $0.28 \mathrm{eV}$. Due to both inversion and time-reversal symmetries of $\mathrm{PtS}_{2} / \mathrm{PtSe}_{2}$, all the bands are doubly degenerate, but each band splits into two energy bands for PtSSe monolayer because of the lack of inversion, which is very useful to allow spin manipulation. Our calculated GGA+SOC gaps of the $\mathrm{PtS}_{2}(1.73 \mathrm{eV})$ and $\mathrm{PtSe}_{2}(1.20 \mathrm{eV})$ monolayers ${ }^{16}$ are very close to the experimental values of $1.60 \mathrm{eV}^{45}$ and $1.20 \mathrm{eV}^{46}$. Thus, it is reasonable to use GGA+SOC to study the electronic structures of PtSSe.

Strain is a very effective way to tune the electronic and phonon properties of $2 \mathrm{D}$ materials, and strain effects on energy band structures and transport properties of TMD and Janus TMD monolayers have been widely investigated $^{16,18,32,44,47,48}$. Here, we examine the effects of biaxial strain on the electronic structures and electronic transport coefficients of PtSSe monolayer. The $a / a_{0}$ is defined to simulate biaxial strain, in which $a$ and $a_{0}$ are the strained and unstrained lattice constant, respectively. The $a / a_{0}<1$ means compressive strain, while 

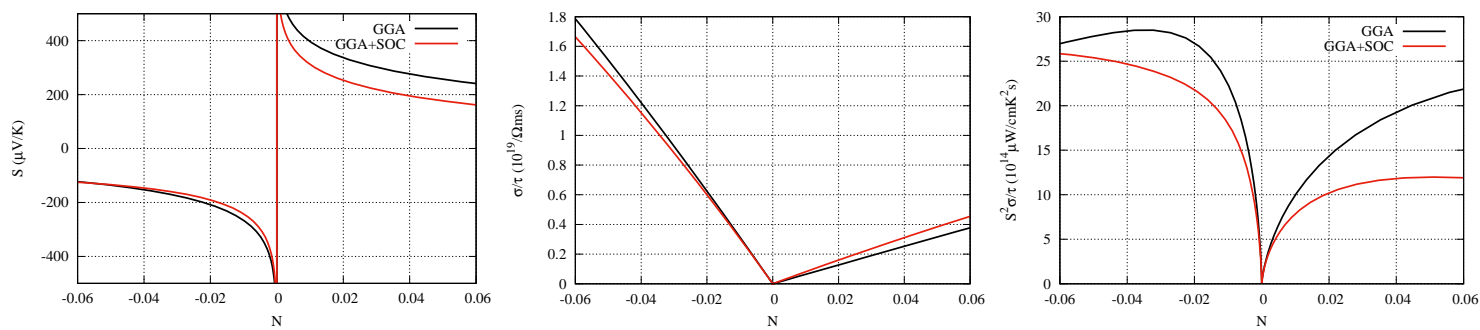

FIG. 5. (Color online) the room-temperature transport coefficients $\left(\mathrm{S}, \sigma / \tau\right.$ and $\left.\mathrm{S}^{2} \sigma / \tau\right)$ of PtSSe monolayer as a function of doping level (N) using GGA and GGA+SOC.

$a / a_{0}>1$ implies tensile strain. The energy band gap (Gap), spin-orbit splitting value at $\Gamma$ point $(\Delta)$, the difference between the Fermi level and the energy of the first valence band at $\Gamma$ point $(\Delta 1)$ and the difference between the second $\mathrm{CBE}$ and CBM (D) as a function of $a / a_{0}$ are plotted in Figure 3, and the related energy band structures with $a / a_{0}$ from 0.94 to 1.06 are shown in Figure 4.

It is clearly seen that the energy band gap firstly increases, and then decreases, when $a / a_{0}$ changes from 0.94 to 1.06. Similar phenomenon can also be found in many TMD and Janus TMD monolayers ${ }^{16,18,32,44}$. With strain from compressive one to tensile one, the $\Delta$ has a slight increase, then a rapid decrease. With increasing strain, the overall trend of $\Delta$ is consistent with one of $1 \mathrm{~T}$ TMD and Janus TMD monolayers, but is opposite to one of $2 \mathrm{H}$ ones $^{18,32,44}$. The strain can tune the positions of CBM and VBM. The compressive one can change the position of CBM from one point along the $\Gamma-\mathrm{M}$ direction to another point along the $\Gamma-\mathrm{K}$ direction, which can be described by D. With $a / a_{0}$ changing from 0.94 to 1.06 , the $\mathrm{D}$ varies from a negative value to a positive one, which means the change of CBM position. The compressive one can also tune the position of VBM from one point along the $\Gamma-\mathrm{K}$ direction to $\Gamma$ point, and can reduce the numbers of VBE from two to one. It is noted that the compressive one can produce the very flat valence band around the $\Gamma$ point, for example one with $a / a_{0}$ being 0.96 . These can be described by $\Delta 1$, and the $\Delta 1=0(\neq 0)$ means the one (two) VBE. In a word, strain can tune the position of VBM (CBM) or the numbers of VBE (CBE), and the similar phenomenon can also be observed in TMD and Janus TMD monolayers ${ }^{16,18,32,44,48}$.

Based on CSTA Boltzmann theory within rigid band approach (RBA), the electronic transport coefficients are investigated. The calculated electrical conductivity $\sigma / \tau$ and electronic thermal conductivity $\kappa_{\mathrm{e}} / \tau$ depend on scattering time $\tau$, while the Seebeck coefficient $\mathbf{S}$ is independent of $\tau$. To simulate the doping effects, simply moving the position of Fermi level within RBA is performed. The $\mathrm{n}(\mathrm{p})$-type doping is achieved with negative (positive) doping levels by shifting the Fermi level into conduction (valence) bands, giving the negative (positive) Seebeck coefficient. For monolayer PtSSe, the room temperature $\mathrm{S}, \sigma / \tau$ and power factor with respect to scattering time $\mathrm{S}^{2} \sigma / \tau$ as a function of doping level (N) are shown in Fig-
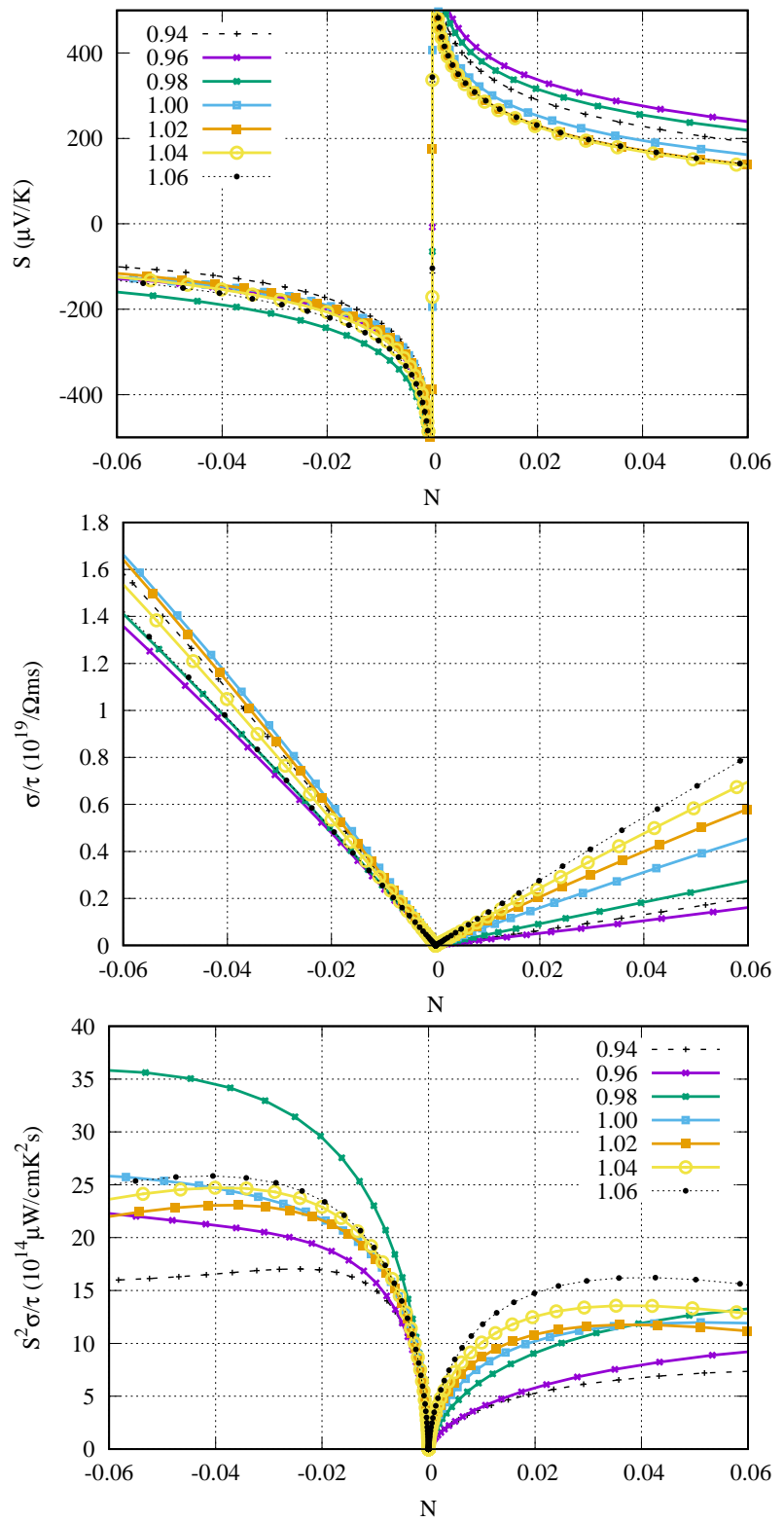

FIG. 6. (Color online) the room-temperature transport coefficients $\left(\mathrm{S}, \sigma / \tau\right.$ and $\left.\mathrm{S}^{2} \sigma / \tau\right)$ of PtSSe monolayer as a function of doping level $(\mathrm{N})$ using GGA+SOC, and the $a / a_{0}$ changes from 0.94 to 1.06 with the interval being 0.02 . 


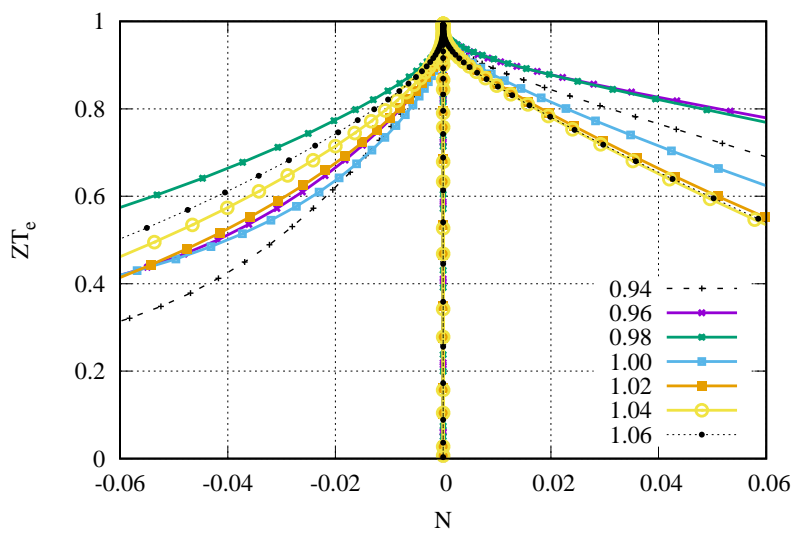

FIG. 7. (Color online) the room-temperature $Z T_{e}$ of PtSSe monolayer as a function of doping level $(\mathrm{N})$ using GGA+SOC, and the $a / a_{0}$ changes from 0.94 to 1.06 with the interval being 0.02 .

ure 5, using GGA and GGA+SOC. For 2D materials, it may be more reasonable to use electrons or holes per unit cell instead of doping concentration, which is described by $\mathrm{N}$, and the minus (positive) values mean $\mathrm{n}$ (p)-type doping. The SOC can induce a detrimental influence on p-type $\mathrm{S}$ of PtSSe monolayer, and produces a slightly reduced effect on $\mathrm{S}$ in n-type doping. These can be explained by considering SOC effects on the bands near the Fermi level. The SOC can remove the band degeneracy near the VBM or CBM, leading to reduced $\mathrm{S}$. The power factor is a comprehensive physical quantity for the electrical performance of thermoelectric materials. Due to the power factor being proportional to $\mathrm{S}$ and $\sigma / \tau$, the SOC has a remarkable detrimental influence on both nand p-type power factor of PtSSe in considered doping range. It is noted that theses results also depend on the strain. When including SOC, if the strength of bands convergence is enhanced, the $\mathrm{S}$ would be improved, producing enhanced power factor. It has been proved in $\mathrm{WX}_{2}\left(\mathrm{X}=\mathrm{S}\right.$, Se and Te) monolayer ${ }^{16}$.

At room temperature, the biaxial strain dependence of $\mathrm{S}, \sigma / \tau$ and $\mathrm{S}^{2} \sigma / \tau$ of PtSSe monolayer are plotted in Figure 6 using GGA+SOC. Because the electronic structures of PtSSe monolayer are sensitively dependent on strain, the complex strain dependence of electronic transport coefficients are observed. With $a / a_{0}$ being 0.98 , the largest $\mathrm{n}$-type $\mathrm{S}$ can be attained among the considered strain points, which can be understood by strain-driven accidental band degeneracies, namely bands convergence. From (d) in Figure 3 and Figure 4 , the D with $a / a_{0}$ being 0.98 is very close to zero, which means that the $\mathrm{CBE}$ along the $\Gamma-\mathrm{K}$ direction and CBM is almost degenerate, producing enhanced S. Among considered doping points, the largest p-type $\mathrm{S}$ can be observed with $a / a_{0}$ being 0.96 , which is due to very flat valence bands around $\Gamma$ point near the Fermi level from Figure 4. For metals or degenerate semiconductors, the $\mathrm{S}$ is can be described by $S=\frac{8 \pi^{2} K_{B}^{2}}{3 e h^{2}} m^{*} T\left(\frac{\pi}{3 n}\right)^{2 / 3}$, in which $m^{*}, \mathrm{~T}$ and

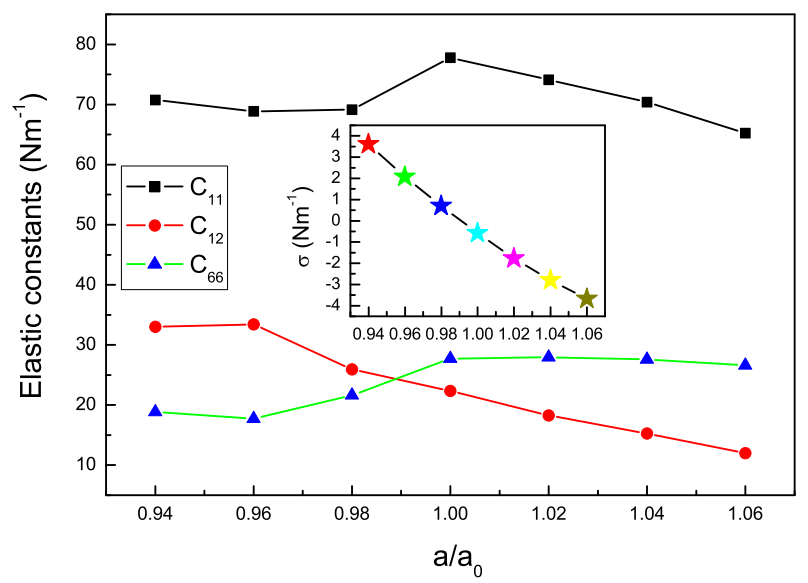

FIG. 8. (Color online) The stress $\sigma$ (inset) and elastic constants $C_{i j}$ vs $a / a_{0}$ from 0.94 to 1.06 for PtSSe monolayer.

$n$ is the effective mass of the carrier, temperature and carrier concentration, respectively. The flat bands can produce very large effective mass of the carrier, and give rise to improved $\mathrm{S}$. In n-type doping, the largest $\mathrm{S}^{2} \sigma / \tau$ can be observed at $a / a_{0}=0.98$ point due to the largest $\mathrm{S}$ among the considered strain points. For p-type doping, at $a / a_{0}=0.96$ point, although the largest $S$ can be attained, the very small $\mathrm{S}^{2} \sigma / \tau$ can be observed, which is because the flat bands lead to very small $\sigma / \tau$. Among the considered strain points, the largest p-type $S^{2} \sigma / \tau$ can be attained at $a / a_{0}=1.06$ point, which is due to the largest $\sigma / \tau$

The $Z T_{e}=S^{2} \sigma T / \kappa_{e}$ can be defined as an upper limit of $Z T$, which neglects the $\kappa_{L}$. At room temperature, the $Z T_{e}$ of Janus PtSSe monolayer as a function of $\mathrm{N}$ with $a / a_{0}$ changing from 0.94 to 1.06 are plotted in Figure 7. It is found that the trend of $Z T_{e}$ as a function of $a / a_{0}$ is consistent with one of S. By the Wiedemann-Franz law: $\kappa_{e}=L \sigma T$ ( $L$ is the Lorenz number $)$, the $\kappa_{\mathrm{e}}$ relates to $\sigma$, and then $Z T_{e}=S^{2} / L$ can be attained. Thus, the strainimproved $S^{2} \sigma / \tau$ caused by enhanced $S$ is beneficial to better $Z T_{e}$. For example, compressive strain can significantly enhance n-(p-)type $Z T_{e}$ of PtSSe monolayer at $a / a_{0}=0.98$ (0.96) point by compressive strain-improved S. It is noted that the very high $Z T$ values of many $2 \mathrm{D}$ materials have been reported ${ }^{49,50}$, which is because the $\kappa_{e}$ are calculated by the Wiedemann-Franz law with $L$ being constant. In our calculations, the $\kappa_{e}$ are used from outputs of BoltzTrap, where the $L$ depends on temperature and doping level.

Finally, to study the mechanical stability of PtSSe monolayer with strain, the elastic constants $C_{i j}$ are calculated as a function of $a / a_{0}$, and are plotted in Figure 8. In considered strain range, they all satisfy the Born criteria of mechanical stability for $2 \mathrm{D}$ hexagonal crystals $^{43}$ : $C_{11}>0$ and $C_{66}>0$. The stress at different $a / a_{0}$ point is also calculated, shown in Figure 8 . In considered strain range, the stress is relatively small from $-3.7 \mathrm{Nm}^{-1}$ to $3.6 \mathrm{Nm}^{-1}$, which can be easily realized experimentally. 

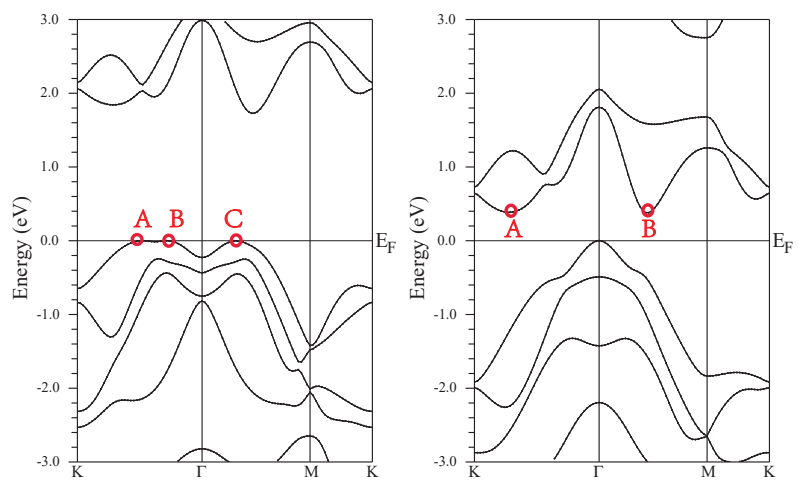

FIG. 9. (Color online)The energy band structures of $\mathrm{PtS}_{2}$ and $\mathrm{PtTe}_{2}$ using GGA+SOC.

That means that strain-induced flat bands and strainimproved $\mathrm{S}$ and $Z T_{e}$ can be easily observed in experiment, which can stimulate further experimental works to synthesize PtSSe monolayer, and then investigate the strain effects on its electronic structures and transport properties.

\section{DISCUSSIONS AND CONCLUSION}

It is very important for investigating the electronic structures of 2D materials to chose appropriate exchange correlation potential. Although the HSE06 can accurately calculate the gaps of many semiconductors, the GGA is more reasonable to study the gaps of $\mathrm{PtS}_{2}$ and $\mathrm{PtSe}_{2}$. The calculated gaps $\left(\mathrm{PtS}_{2}(1.73 \mathrm{eV})\right.$ and $\mathrm{PtSe}_{2}$ $(1.20 \mathrm{eV}))^{16}$ with GGA are more close to the experimental values $\left(\mathrm{PtS}_{2}(1.60 \mathrm{eV}) \text { and } \mathrm{PtSe}_{2}(1.20 \mathrm{eV})\right)^{45,46}$ than ones with HSE06 $\left(\mathrm{PtS}_{2}(2.63 \mathrm{eV})\right.$ and $\mathrm{PtSe}_{2}(1.74$ $\mathrm{eV}))^{42}$. Thus, in this work, the GGA is used to study the electronic structures and transport coefficients of Janus PtSSe monolayer. For TMD and Janus TMD monolayers, the SOC produces a remarkable influence on their electronic structures, and especially transport coefficients ${ }^{16,32}$. The SOC not only can reduce the power factor of TMD and Janus TMD monolayers, but can also obviously improve one, which depends that the strength of bands convergence will be strengthened or weakened caused by SOC.
Strain can tune electronic structures, topological and transport properties of $2 \mathrm{D}$ materials by changing the distance between the atoms. Strain can tune the positions of VBM and CBM, the numbers of VBE and $\mathrm{CBE}$ and the strength of bands convergence. For PtSSe monolayer, in n-type doing, a very small compressive strain $\left(a / a_{0}=0.98\right)$ can induce the bands convergence, which gives rise to improved S. For p-type doping, a small compressive strain $\left(a / a_{0}=0.96\right)$ can lead to flat bands around the $\Gamma$ point, inducing the enhanced $S$. In fact, in unstrained $\mathrm{PtS}_{2}$ and $\mathrm{PtTe}_{2}$, band convergence can be realized, and their energy band structures using GGA+SOC are plotted in Figure 9. For $\mathrm{PtS}_{2}$, the A, B and $\mathrm{C}$ points in the valence bands have almost the same energy, producing the valence band convergence. For $\mathrm{PtTe}_{2}$, in the conduction bands, the energy difference between the A and $\mathrm{B}$ points is very small, giving rise to conduction band convergence.

In summary, the strain dependence of electronic structures and transport coefficients of Janus PtSSe monolayer are systematically studied from the reliable first-principle calculations. It is found that the SOC can produce important effects on energy band structures and electronic transport coefficients of Janus PtSSe monolayer. Calculated results show that compressive strain can change the positions of CBM and VBM of PtSSe monolayer, and can induce flat valence bands around the $\Gamma$ point. These changes can lead to improved S, and give rise to better $Z T_{e}$. In the considered strain range, the PtSSe monolayer is mechanically stable by calculated elastic constants, which satisfy the mechanical stability criteria. Our works can stimulate further experimental works to synthesize PtSSe monolayer, and will motivate farther studies of electronic transports of other Janus monolayers.

\section{ACKNOWLEDGMENTS}

This work is supported by the National Natural Science Foundation of China (Grant No. 11404391). We are grateful to the Advanced Analysis and Computation Center of China University of Mining and Technology (CUMT) for the award of CPU hours to accomplish this work.
1 K. S. Novoselov et al., Science 306, 666 (2004).

2 J. P. Ji, X. F. Song, J. Z. Liu et al., Nat. Commun. 7, 13352 (2016).

3 S. Balendhran, S. Walia, H. Nili, S. Sriram and M.Bhaskaran, small 11, 640 (2015).

${ }^{4}$ S. L. Zhang M. Q. Xie, F. Y. Li, Z. Yan, Y. F. Li, E. J. Kan, W. Liu, Z. F. Chen, H. B. Zeng, Angew. Chem. 128, 1698 (2016).

${ }^{5}$ M. Chhowalla, H. S. Shin, G. Eda, L. J. Li, K. P. Loh and
H. Zhang, Nature Chemistry 5, 263 (2013).

${ }^{6}$ R. X. Fei, W. B. Li, J. Li and L. Yang, Appl. Phys. Lett. 107, 173104 (2015).

7 L. D. Hicks and M. S. Dresselhaus, Phys. Rev. B 47, 12727 (1993).

${ }^{8}$ L. D. Hicks and M. S. Dresselhaus, Phys. Rev. B 47, 16631(R) (1993).

9 Y. Pei, X. Shi, A. LaLonde, H. Wang, L. Chen and G. J. Snyder, Nature 473, 66 (2011). 
10 W. Huang, H. X. Da and G. C. Liang, J. Appl. Phys. 113, 104304 (2013).

11 G. P. Li, G. Q. Ding and G. Y. Gao, J. Phys.: Condens. Matter 29, 015001 (2017).

12 G. Qin, Z. Qin, W. Fang, L. Zhang, S. Yue, Q. Yan, M. $\mathrm{Hu}$ and G. Su, Nanoscale 8, 11306 (2016).

13 L. M. Sandonas,D. Teich, R. Gutierrez, T. Lorenz, A. Pecchia, G. Seifert and G. Cuniberti, J. Phys. Chem. C 120, 18841 (2016).

14 S. D. Guo and Y. H. Wang, J. Appl. Phys. 121, 034302 (2017).

15 D. C. Zhang, A. X. Zhang, S. D. Guo and Y. F. Duan, RSC Adv. 7, 24537 (2017).

16 S. D. Guo and J. L. Wang, Semicond. Sci. Tech. 31, 095011 (2016).

17 H. Y. Lv, W. J. Lu, D. F. Shao, H. Y. Lub and Y. P. Sun, J. Mater. Chem. C 4, 4538 (2016).

18 S. D. Guo, J. Mater. Chem. C 4, 9366 (2016).

19 G. P. Li, G. Q. Ding and G. Y. Gao, J. Phys.: Condens. Matter 29, 015001 (2017).

${ }^{20}$ H. K. Liu, G. Z. Qin, Y. Lin and M. Hu, Nano Lett. 16, 3831 (2016).

21 A. Shafique and Y. H. Shin, Phys. Chem. Chem. Phys. 19, 32072 (2017).

${ }^{22}$ L. Lindsay, Wu Li, J. Carrete, N. Mingo, D. A. Broido and T. L. Reinecke, Phys. Rev. B 89, 155426 (2014).

23 A. Y. Lu, H. Y. Zhu, J. Xiao et al., Nature Nanotechnology 12, 744 (2017).

${ }^{24}$ L. Dong, J. Lou and V. B. Shenoy, ACS Nano 11, 8242 (2017).

25 W. J. Yin, B. Wen, G. Z. Nie and X. L. Wei and L. M. Liu, J. Mater. Chem. C 61693 (2018).

26 Y. C. Cheng, Z. Y. Zhu, M. Tahir et al., Europhys. Lett. 102, 57001 (2013).

27 X. C. Ma, X. Wu, H. D. Wang and Y. C. Wang, J. Mater. Chem. A 6, 2295 (2018).

28 R. Peng, Y. D. Ma, B. B. Huang and Y. Dai, J. Mater. Chem. A 7, 603 (2019).

29 F. P. Li, W. Wei, P. Zhao, B. B. Huang and Y. Dai, J. Phys. Chem. Lett. 8, 5959 (2017).

30 S. D. Guo, Phys. Chem. Chem. Phys. 20, 7236 (2018).

31 S. D. Guo, Y. F. Li and X. S. Guo, Comp. Mater. Sci. 161,
16 (2019).

32 S. D. Guo and J. Dong, Semicond. Sci. Tech. 33, 085003 (2018).

33 P. Hohenberg and W. Kohn, Phys. Rev. 136, B864 (1964); W. Kohn and L. J. Sham, Phys. Rev. 140, A1133 (1965).

${ }^{34}$ P. Blaha, K. Schwarz, G. K. H. Madsen, D. Kvasnicka and J. Luitz, WIEN2k, an Augmented Plane Wave + Local Orbitals Program for Calculating Crystal Properties (Karlheinz Schwarz Technische Universität Wien, Austria) 2001, ISBN 3-9501031-1-2

35 J. P. Perdew, K. Burke and M. Ernzerhof, Phys. Rev. Lett. 77, 3865 (1996).

36 A. H. MacDonald, W. E. Pickett and D. D. Koelling, J. Phys. C 13, 2675 (1980).

37 D. J. Singh and L. Nordstrom, Plane Waves, Pseudopotentials and the LAPW Method, 2nd Edition (Springer, New York, 2006).

38 J. Kunes, P. Novak, R. Schmid, P. Blaha and K. Schwarz, Phys. Rev. B 64, 153102 (2001).

39 D. D. Koelling, B. N. Harmon, J. Phys. C: Solid State Phys. 10, 3107 (1977).

40 G. K. H. Madsen and D. J. Singh, Comput. Phys. Commun. 175, 67 (2006).

41 X. F. Wu, V. Varshney et al., Chem. Phys. Lett. 669, 233 (2017).

42 W. L. Tao, Y. Mu, C. E. Hu, Y. Cheng and G. F. Ji, Philosophical Magazine, 99, 1025 (2019).

43 R. C. Andrew, R. E. Mapasha, A. M. Ukpong and N. Chetty, Phys. Rev. B 85, 125428 (2012).

44 S. D. Guo, Comp. Mater. Sci. 123, 8 (2016).

${ }^{45}$ Y. Zhao, J. Qiao, P. Yu, Z. Hu, Z. Lin, S.P. Lau, Z. Liu, W. Ji and Y. Chai, Adv. Mater 28, 2399 (2016).

46 Y. Wang, L. Li, W. Yao et al., Nano Lett. 15, 4013 (2015).

47 E. Scalise, M. Houssa, G. Pourtois, V. Afanas'ev and A. Stesmans, Nano Res. 5, 43 (2012).

48 D. Qin, X. J. Ge, G. Q. Ding, G. Y. Gao and J. T. Lv, RSC Adv. 7, 47243 (2017).

49 L. Cheng, H. J. Liu, X. J. Tan, J. Zhang, J. Wei, H. Y. Lv, J. Shi and X. F. Tang, J. Phys. Chem. C 118, 904 (2014).

${ }^{50}$ F. Q. Wang, S. Zhang, J. Yu and Q. Wang, Nanoscale 7, 15962 (2015). 